\title{
Clinical Trial Evaluating an Online Cognitive Dysfunction Evaluation System for Stroke Patients
}

\author{
In-Hee Lee, PT, PhD ${ }^{1)}$, SAng-Young PARk, PT, MSC ${ }^{2)}$, \\ Chang-SiK Son, $\mathrm{PhD}^{3)}$, Yoon-Nyun Kim, MD, $\mathrm{PhD}^{4)}$ \\ 1) Department of Physical Medicine and Rehabilitation, Dongsan Hospital, Keimyung University \\ 2) Graduate School of Physical Therapy, Daegu University \\ 3) Department of Medical Informatics, School of Medicine, Keimyung University \\ 4) Department of Internal Medicine, School of Medicine, Keimyung University: Dalsung-Ro, Jung-Gu, \\ Daegu, 700-712 Korea.TEL: +82 53-250-7432,FAX:+82 53-580-3745,E-mail:ynkim@dsmc.or.kr
}

\begin{abstract}
Purpose] The purpose of this study was to verify the validation and usefulness of an online cognitive examination system to measure the degree of cognitive deficits of stroke patients. [Subjects] To verify the usefulness of the online examination system, thirty-one stroke patients and twenty normal, healthy volunteers as an agematched control group were recruited. [Methods] The thirty-one stroke patients were classified into three groups according to the severity of their cognitive dysfunction. To evaluate the reliability of the online system we compared the results of our system with the results of the Korean version of the Mini-Mental Status Examination (MMSE-K). [Results] There were significant correlations between the results of the evaluation by the online-based system and those of MMSE-K, and there were also significant differences between patient subgroups and the control group. [Conclusions] We have investigated the usefulness of an online evaluation system and verified its effectiveness in the screening of patients for cognitive dysfunction.

Key words: Cognitive dysfunction, Online examination system, stroke
\end{abstract}

(This article was submitted Nov. 22, 2011, and was accepted Jan. 26, 2012)

\section{INTRODUCTION}

Stroke can be a major cause of impaired cognitive ability. Cognitive issues cause impairments in attention, memory, perception and the ability to perform activities of daily living (ADL). Thus it is important to accurately evaluate the degree of cognitive impairment in these individuals in addition to developing an effective method of measuring cognitive dysfunction.

Cognition can be defined very broadly as the acquisition and use of knowledge $\rrbracket$. It usually refers to the ability of the brain to process, store, retrieve and manipulate information 1 . Based on this definition, cognitive examinations are assessments of the cognitive capabilities of patients. Moreover, they are widely used as standard practice in a number of professional fields such as the medical, educational and industrial areas. Traditionally, the most common use of cognitive examinations has been in clinical practice and in research examining neurological and neuropsychiatric disorders.

Various tests have been proposed to help evaluate cognitive ability including the Halstead-Raintan test, the Luria-Nebraska test, the revised Wechsler adult intelligence scale, etc ${ }^{3-5}$. However, these methods have the following drawbacks: i) the examinations are time-consuming, ii) errors frequently occur in scoring 9 , iii) the intervention of the evaluator can affect the results of the cognitive exami- nation $\AA$, iv) storage problems occur because these tests are based on paper and pencil, and v) there is criticism that patients are not tested in a practical manner. Because of these issues, many of these methods are not widely used. The most commonly used cognitive dysfunction screening tool in medicine is the mini-mental state examination (MMSE) which was developed by Folstein et al., in 1975 p. This examination tool takes about 10 minutes.

To help overcome the drawbacks of the conventional tests, various computer-based examination tools including the Cambridge Automated Neuropsychological Test Battery (CAN-TAB) 3 , MicroCog (MC) etc-11. have been proposed. The benefits of computer-based examinations are as follows: i) they make collection and storage of test data easy, ii) they have high accuracy and reliability in relation to the time spent on the test $t^{12-14}$, and iii) they reduce the intervention of the evaluator. However, these systems require specialized hardware and software and the VR-based system requires various equipment in addition to causing cyber sickness, vomiting and disorientation problems 11 . These drawbacks make them unsuitable for clinics on a restrictive budget and for patients who are assessed at multiple departments including neuropsychiatry, neurology and rehabilitation clinics.

To overcome these drawbacks and to construct a system which is free from the constraints of the time and place of the examination, an online cognitive examination system may 
Table 1. General characteristics of the subjects

\begin{tabular}{lllll}
\hline & Normal control & \multicolumn{4}{l}{ Patient groups $(\mathrm{n}=31)$} \\
\cline { 3 - 5 } & $(\mathrm{n}=20)$ & Group I $(\mathrm{n}=20)$ & Group II $(\mathrm{n}=6)$ & Group III $(\mathrm{n}=5)$ \\
\hline Age (years) & $55.2 \pm 4.4$ & $55.5 \pm 10.4$ & $57.5 \pm 16.9$ & $63.2 \pm 10.1$ \\
Gender (male:female) & $12: 8$ & $15: 5$ & $5: 1$ & $4: 1$ \\
Hemiparesis side (left:right) & & $11: 9$ & $3: 3$ & $3: 2$ \\
MMSE-K & $30.00 \pm 0.00$ & $28.25 \pm 1.71$ & $22.17 \pm 1.33$ & $15.60 \pm 2.30$ \\
\hline
\end{tabular}

※ Values are mean \pm standard deviation.

be beneficial. The merits of the online system include the ability to collect large amounts of data, minimal constraints of time and place, and reduced cost, because they do not need additional hardware and/or software equipment. Therefore we have compiled an online cognitive examination system and verified it's usefulness and effectiveness, by comparing it with the Korean version of Mini-Mental Status Examination $(\mathrm{MMSE}-\mathrm{K}){ }^{15}$.

\section{SUBJECTS AND METHODS}

To verify our online-based cognitive examination system, fifty-two patients with stroke were recruited. However, twenty-one patients were excluded because they did not give their informed consent to this study had other neurologic illnesses. Therefore, fifty-one participants (thirty-one post-stroke patients and twenty normal healthy volunteers) were recruited and enrolled in this study after providing their informed consent in accordance with the ethical standards of the Declaration of Helsinki. Out of the thirty-one post stroke patients, seventeen had cerebral infarction and thirteen had cerebral hemorrhage. All the patients were recruited from a rehabilitation hospital in Daegu, Korea.

All of the participants performed the MMSE-K examination and the thirty-one post stroke patients were classified into three groups according to MMSE-K score: Group I of 20 patients ( $\geq 24)$, Group II of 6 patients (20 -23$)$, and Group III of 5 patients $(\leq 19)^{16}$. The age range of the participants was 34-97, with a mean of 56.5 years. Participants were screened for history of neurologic illness or head injury, developmental disorders, aphasia, dyslexia and the ability to stay in a sitting posture. Moreover, twenty normal, healthy participants with no history of neurologic disorders were recruited as a control group. The mean age of control group was 55.5 years (Table 1 ).

To simplify the examination, we designed four multiple choice types of questions which show the most sensitive reactions in neuropsychology 17 . To minimize error due to individual language skills, all test stimuli consisted of nonverbal communication. The online-based examination consisted of four sections: Orientation, Attention, Memory, and Executive function. Moreover two language questions were included in each of the Attention and Executive function sections (Fig. 1). The characteristics and explanation of each section are as follows.

The orientation section consisted of ten questions. We designed questions with various figures such as a hospital,

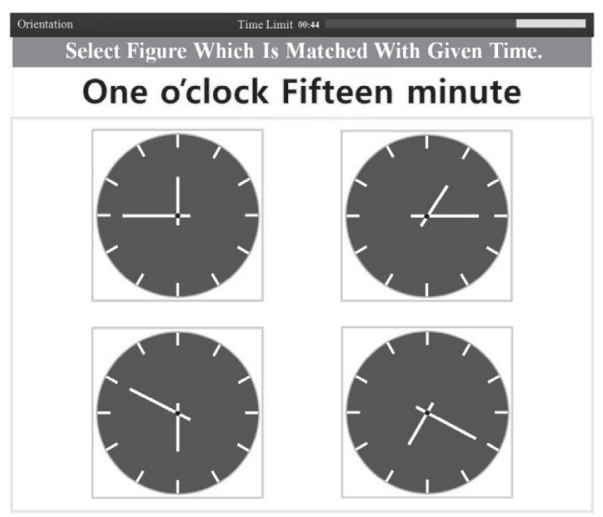

(a) Example of orientation; select a figure which is matched given time

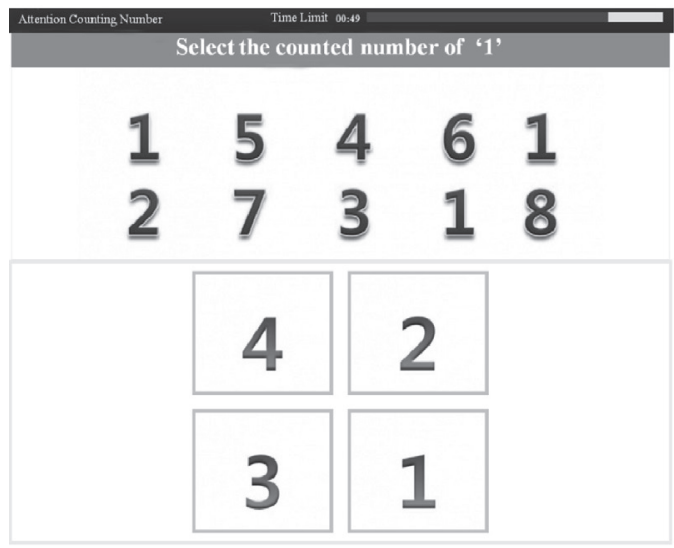

(b) Example of attention; select the counted number of "1"

Fig. 1. Examples of questions of the online-based system

toilet, season, person with a specific job, etc. To evaluate attention, we designed questions that included finding the number of specific digits and/or symbols among a display and finding specific information through a question. The attention section consisted of eight questions. Memory is defined as the ability to recall and register past information. The online system showed a figure for ten seconds to evaluate the short-term recall ability of the subjects. After the ten seconds, the figure disappeared and participants were requested to answer the questions. The memory section consisted of ten questions. Executive function is defined as the ability to control and/or manage a given situation such as planning, decision making, problem solving, etc. Therefore, 
Table 2. Comparison of test categories between the patient groups and the control group

\begin{tabular}{lclll}
\hline \multirow{2}{*}{ Control Group $(\mathrm{n}=20)$} & \multicolumn{4}{l}{ Patient Groups $(\mathrm{n}=31)$} \\
\cline { 3 - 5 } & & Group I $(\mathrm{n}=20)$ & Group II $(\mathrm{n}=6)$ & Group III $(\mathrm{n}=5)$ \\
\hline Orientation & $10.00 \pm 0.00$ & $9.65 \pm 0.75$ & $8.67 \pm 0.52^{* \dagger}$ & $7.20 \pm 1.30^{* \dagger *}$ \\
Language & $3.95 \pm 0.22$ & $3.55 \pm 0.83$ & $2.50 \pm 1.05^{*}$ & $2.00 \pm 1.87^{* \dagger}$ \\
Concentration & $7.50 \pm 1.00$ & $7.10 \pm 1.12$ & $5.00 \pm 1.26^{* \dagger}$ & $3.75 \pm .126^{* \dagger}$ \\
Memory & $9.40 \pm 0.68$ & $8.65 \pm 1.35$ & $8.00 \pm 1.67$ & $6.00 \pm 1.22^{* \dagger}$ \\
Execution & $7.70 \pm 0.57$ & $6.35 \pm 1.31^{*}$ & $4.00 \pm 1.10^{* \dagger}$ & $3.40 \pm 0.55^{* \dagger}$ \\
Total Score & $38.45 \pm 1.67$ & $35.30 \pm 3.61^{*}$ & $28.17 \pm 3.06^{* \dagger}$ & $22.20 \pm 2.17^{* \dagger}$ \\
\hline
\end{tabular}

※ p-values were calculated by ANOVA with post-hoc Scheffe and Dunnett T3 test. ${ }^{*} \mathrm{p}<0.05$ vs. control group; $\left\lceil\mathrm{p}<0.05\right.$ vs. group I; ${ }^{\ddagger} \mathrm{p}<0.05$ vs. group II.

we designed various situations (e.g. cooking, finding proper tools, etc.) to evaluate ability. The execution function section consisted of eight questions. The Language section of 4 questions consisted of finding incorrectly spelled words.

Moreover, to minimize the intervention of the evaluator, we imposed a one-minute time limit on each question; after one minute, the system automatically switched to the next question. The examination was taken section by section in order of orientation, attention, memory and executive function. To eliminate any learning effect, the questions were presented randomly.

The online system is hosted on Apache, with My SQL, PHP, and Xpress Engine. The system consists of an administrator mode and user mode. In administrator mode, question, subject, figure, and user managements are possible. For the user, instructions and practice problems are provided. Therefore, before taking the cognitive examination, it is possible for users to obtain assistance, and instructions, and to practice problems.

The examination was performed at a quiet place without intervention. To learn how to take the examination and to solve the problems, participants were requested to solve practice problems before taking the actual examination. Moreover, during the practice test, if participants required further clarification of instructions, experts gave further instruction about the online system. After the practice test, fifty-one participants took the examination and retests were performed after two days to evaluate the reliability of the online system.

The examination results of the online system were compared with the results of the MMSE-K. The results of the MMSE-K were scored as follows: a maximum of ten points for orientation, six points for registration and recall, five points for attention, six points for execution, and three points for language. A one-way ANOVA was performed to analyze the differences between the results of the control group and patient groups in the online cognitive examination. Pearson's correlation coefficient was used to analyze the correlation between the online system and MMSE-K, and both a Spearman rho analysis and Cronbach's analysis were performed to evaluate the reliability of the online cognitive examination system and the internal consistency of the categories.

\section{RESULTS}

Table 2 compares the online cognitive examination system results of the test groups. The average scores of the control group, patient group I, group II and group III were $38.45,35.30,28.17$ and 22.20 , respectively. The execution score of the control group was significantly higher than of the patient group I $(\mathrm{p}<0.05)$. Orientation, language function, attention, and execution scores of the control group were significantly higher than those of patient group II $(p<0.05)$. The scores of all categories for the control group were significantly higher than those of patient group III $(p<0.05)$. Orientation, attention and execution scores of patient group I were significantly higher than those of patient group II $(p<0.05)$. The scores of all categories for patient group I were significantly higher than those of patient group III $(p<0.05)$. The orientation score of patient group II was significantly higher than that of patient group III $(\mathrm{p}<0.05)$.

Table 3 shows the correlation results. Significant correlations were found between each category score of online cognitive function test and the respective MMSE-K category score $(p<0.01)$. Regarding the test-retest scores shown in Table 4, Test-retest was not statistically different. The Spearman rho coefficient was 0.720 .

Table 5 shows the results of internal consistency according to the categories of the online system. Language $(0.750)$ and execution (0.710) showed high internal consistencies, with values over 0.7. Moreover, memory, concentration, and orientation categories had values of over 0.6 . That is, all of the topics satisfied the minimum criterion $(>0.5)$ of internal consistency.

\section{DISCUSSION}

Various studies have evaluated the effectiveness of computerized cognitive systems, based on the response time. Conventional computerized tests of cognitive function have been based on written tests or need the intervention of an evaluator, and they have difficulties evaluating the cognitive dysfunction of elderly patients with physical limitations, because even elderly people, who do not have cognitive impairment, react slowly due to senility. Moreover, conventional computerized tests through personal interviews are time consuming, expensive and require many profes- 
Table 3. Correlation between categories of MMSE-K and the online examination system

\begin{tabular}{ll}
\hline Index & Correlation Coefficient \\
\hline Orientation & $0.743^{*}$ \\
Attention & $0.720^{*}$ \\
Memory & $0.685^{*}$ \\
Execution & $0.397^{*}$ \\
Language & $0.419^{*}$ \\
Total & $0.882^{*}$ \\
${ }^{*} \mathrm{p}<0.005$ &
\end{tabular}

Table 4. Reliability of the test and retest scores

\begin{tabular}{lll}
\hline Test & Retest & Coefficient \\
\hline $30.75 \pm 5.83$ & $32.35 \pm 6.89$ & 0.720 \\
\hline
\end{tabular}

Table 5. Internal consistency according to category

\begin{tabular}{ll}
\hline Indexes & Cronbach's Alpha \\
\hline Orientation & 0.635 \\
Language & 0.750 \\
Concentration & 0.648 \\
Memory & 0.692 \\
Execution & 0.710 \\
\hline
\end{tabular}

sionals. Therefore, performing research with conventional computerized tests is costly and time consuming. The disadvantages of conventional tests are that the face-to-face testing environment exposes individuals to social pressure or embarrassment; in addition, the conventional tests do not accurately reflect the cognitive functions used in daily life. We could overcome these problems through the introduction of an online test that enables objective evaluation of individuals' cognitive dysfunction. In this way, social pressure and embarrassment that might be present in faceto-face testing can be reduced 18,19 . The intervention of an evaluator may also induce errors, affecting the test results.

An online system has many advantages: i) the elimination of the need for special hardware and software on a local computer2, ii) repeated use with no additional expense, which is ideal for testing very large samples 21, iii) data collection with less error, because it does not require human transcription and data entries 19,21 , and iv) it provides a method of collecting very large data samples quickly and easily 2 .

In addition, paper-based neuropsychological evaluations do not accurately reflect cognitive functions used in daily life. We could overcome this problem through task-solving of situations encountered in daily living. Moreover, we expect that online systems will simplify many problems associated with serial assessment and facilitate the spread of the clinical implementation of such online screening tools. The advantages of the online-based system can be summarized as follows: i) it does not require specific hardware and/or software installation, ii) clinicians benefit from the continuity of record keeping, iii) it is possible to compare the prognosis of patients, based on the recorded information, and iv) telemedicine consultations are possible for case review, allowing many professionals to view and discuss findings simultaneously.

In this study, we verified the usefulness and effectiveness of an online system for evaluating cognitive dysfunction by comparing the online system and MMSE-K results of fifty-one participants (thirty-one post-stroke patients and twenty normal healthy people). The results show that there were no significant differences between the online system and MMSE-K, and the online system showed high test-retest reliability. These results are in agreement with the report by Luce who showed that online test systems are useful and effective in various fields 2 .

The computer skills of an individual and familiarity with the computer system can affect the examination score. Although, we did consider computer experience, our subject sample was too small to be a representative sample. Therefore, more studies are needed to evaluate the effect on the results of computer experience of patients with various diseases, including traumatic brain injury (TBI), Alzheimer's, dementia, multiple sclerosis, etc.

\section{REFERENCES}

1) Neisser U: Cognition and Reality, San Fransisco: W.H. Freeman, 1976.

2) Prigatano GP, Fordyce DJ: Cognitive dysfunction and psychological adjustment after brain I jury, In: GP Prignatano, ed. Neuropsychological Rehabilitation after Brain Injury. Baltimore: Johns Hopkins University Press, 1986.

3) Allard G, Butler J, Faust D, et al.: Errors in hand scoring objective personality tests: The case of the Personality Diagnostic QuestinaireReivsed(PDQ-R). Prof Psychol Res Pr, 1995, 26: 304-308. CrossRef

4) Moon GW, Blakey WA, Gosuch RL, et al.: Frequent WAIS-R administration errors: An ignored sources of inaccurate measurement. Prof Psychol Res Pr, 1991, 22: 256-258. [CrossRef]

5) Collingwood LM, Harrel EH: Performance of psychotic and substance abuse patients with or without head injury on the Halstead-reitan battery. Appl Neuropsychol, 1999, 6: 88-95. [Medline] [CrossRef]

6) Folstein MF, Folstein SE, McHugh PR: "Mini-mental state". A practical method for grading the cognitive state of patients for the clinician. J Psychiatr Res, 1975, 12: 189-198. [Medline] [CrossRef]

7) Morris RG, Evenden JL, Sahakian BJ, et al.: Computer-aided assessment of dementia: Comparative studies of neuropsychological deficits in Alzheimer-type dementia and Parkinson's disease. In: Stahl SM, Iversen SD, Goodman EC(Eds.), Cognitive neurochemistry Oxford: Oxford University Press, 1986, pp 21-36.

8) Devivo K, Rothland J, Price L, et al.: Computerized assessment of arithmetic computation skills with MicroCog. J Int Neuropsychol Soc, 1997, 3: 199-200. [Medline

9) Golden CJ, Hammeke TA, Prish AD: Luria-Nebraska Neuropsychological Battery: A Manual for clinical and experimental Uses. Los Angeles: University of Nebraska Press, 1980.

10) David W: Wechsler memory scale-revised manual, 1st ed, San Antonio: Hacourt Brace Jovanovich, 1987.

11) Regan EC, Price KR: The frequency occurrence and severity of side-effects of immersion virtual reality. Aviat Space Environ Med, 1994, 65: 527-530. Medline

12) Lauren P, Jorgensen T: Computerized neuropsychological test system: In Neurobehavioral Method in Occupational and Environmental health. WHO Environmental Health Document 3, Cophenhagen, 1985.

13 Nell V: Cross-Cultural Neuropsychological Assessment: Theory and Practice. New Jersy: Lawrence Erbaum Associates, 1999.

14) Paul RH, Lawrence J, Williams LM, et al.: Preliminary validity of "IntegNeuro": a new computerized battery of neurocognitive tests. Int J Neurosci, 2005, 115: 1549-1567. Medline] [CrossRef

15 Kwon YC, Park JH: Korean version of mini-mental state examination(MMSE-K). J Korean Neurol Assoc, 1989, 1: 123-135.

16) Zec RF: Neuropsychological functioning in Alzheimer's disease. In: Parks 
RW, Zec RW, Wilson RS: Neuropsychology of Alzheimer's disease and other dementia. 1st ed. New York: Oxford University Press, 1993, pp 3-80.

17) Maddocks D, Sailing M: Neuropsychological deficits following concussion. Brain Inj, 1996, 10: 99-104. [Medline] [CrossRef]

18) Birnbaum MH: Human research and data collection via the internet. Annu Rev Psychol, 2004, 55: 803-832. [Medline] CrossRef

19) Kraut R, Olson J, Banaji M, et al.: Psychological Research Online. Am Psychol, 2004, 59: 105-117. [Medline] [CrossRef

20) Silverstein SM, Berten S, Olson P, et al.: Development and validation of a world-wide-web-based neurocognitive assessment battery: webneuro. Behav Res Methods, 2007, 39: 940-949. [Medline] [CrossRef

21) Naglieri JA, Drasgow F, Schmit M, et al.: Psychological testing on the internet: new problems, old issues. Am Psychol, 2004, 59: 150-162. Medline CrossRef

22) Luce KH, Winzelberg AJ, Das S, et al.: Reliability of self-report: Paper versus online administration. Comput Human Behav, 2007, 23: 1384 1389. CrossRef 